\title{
An alternative theory of imprecision *
}

\author{
Stephanie Solt \\ Centre for General Linguistics (ZAS)
}

\begin{abstract}
Numerical expressions are often used imprecisely or approximately. This paper defends a novel analysis of numerical imprecision based on the notion of scale granularity, construed here in terms of sets of alternatives. I apply this approach to account for previously unnoticed facts relating to the interaction of (im)precision and comparison, in particular the necessarily precise interpretation of measure expressions in comparatives, and the negative polarity status of overt approximators in comparatives (e.g., Mabel owns *(no) more than about one hundred sheep).
\end{abstract}

Keywords: Approximation, imprecision, granularity, comparatives, alternatives

\section{Introduction}

Numerical expressions with (potentially) precise meanings can be-and often areused imprecisely or approximately.

The basic facts relating to numerical imprecision are well established. First, round numbers such as those in (1) allow or even favor approximate readings (see especially Krifka 2002, 2009). Thus (1a) might be felicitously used to describe a situation where Mabel had a couple more or fewer than exactly 100 sheep; (1b) an arrival that was actually closer to 3:02; (1c) a meeting that was a bit longer or shorter than 45 minutes; and (1d) a rope whose length fell within some range around $50 \mathrm{~m}$.
a. Mabel owns one hundred sheep.
b. Jane arrived at three o'clock.
c. The meeting lasted forty-five minutes.
$\mathrm{d}$. The rope is fifty meters long.

By contrast, non-round numbers, such as those in the corresponding examples in (2), are necessarily interpreted (more) precisely:

* I would like to express my gratitude to the reviewers and audience of SALT 24, and to the audiences at the ZAS Berlin and CRISSP, where previous versions of this work were presented. Particular thanks are due to Chris Cummins, Chris Kennedy, Marie-Christine Meyer, Hazel Pearson, Ciyang Qing, Uli Sauerland and Carla Umbach for comments and suggestions that have found their way directly into the analysis. Thanks also to Nadja Reinhold for assistance with the corpus analysis. All errors are of course my own. Work on this project was supported by the DFG under grant 1157/1-1. 
Imprecision

(2) a. Mabel owns ninety nine sheep.

b. Jane arrived at three-oh-one.

c. The meeting lasted forty-three minutes.

$\mathrm{d}$. The rope is fifty-one meters long.

Finally, (im)precision can be regulated overtly by means of modifiers such as about, approximately and exactly, a class which, following Sauerland \& Stateva (2011), I refer to as approximators:

(3) a. Mabel owns about one hundred sheep.

b. Jane arrived at approximately three o' clock.

c. The meeting lasted roughly forty-five minutes.

$\mathrm{d}$. The rope is exactly fifty meters long.

This paper investigates some largely unnoticed facts relating to numerical imprecision as it interacts with the expression of comparison. It will be seen that these data are problematic for existing accounts of imprecision, but point the way to a more empirically adequate approach. I propose an analysis based on scale granularity, here given a novel implementation in terms of sets of alternatives.

The structure of the paper is as follows: Section 2 introduces the linguistic data that are the primary focus. Sections 3 and 4 examine the two leading semantic/pragmatic approaches to imprecision, and demonstrate issues they each face in accounting for these (and other) facts. Section 5 presents the core of the present proposal, and Section 6 augments this with a theory of structurally determined alternatives (per Katzir 2007) to complete the analysis of the relevant data. Finally, Section 7 concludes.

\section{Approximation and comparison}

Despite there being considerable literature on the semantics of imprecision and approximators, their availability in complex numerical expressions has received little attention. Here I focus on two patterns involving the comparative.

a) Measure expressions in comparatives are interpreted precisely. Whereas the round numbers in (1) allow approximate interpretations, those same values in the comparatives in (4) and (5) can only be interpreted precisely:

(4) Mabel owns more than one hundred sheep.

(5) The rope is more than fifty meters long. 
That is, while (1a) might in the appropriate context be felicitously used in the case that Mabel owned 98 sheep, (4) cannot possibly be used to describe a situation where she owned 99, even though 99 is greater than a value that, if we were speaking imprecisely, would count as 'one hundred'. Nor would it seem that there is a context where (4) would be infelicitous or false if the number of sheep owned were 101, though this is also a value that would fall within the range compatible with one hundred on its approximate interpretation. ${ }^{1}$

Judgments relating to (5) are somewhat more complex. Similarly to the previous example, (5) could never be applied to a rope 49 meters in length, even in a context where a 48-meter rope could be described as fifty meters long. The second part of the diagnostic is however trickier: Surely if the rope were just a couple of centimeters longer than $50 \mathrm{~m},(5)$ might be judged false. But I believe that there is nonetheless a difference relative to an unmodified example such as those in (1). In (1d), it would seem that the context might favor a quite rough interpretation of fifty meters (say, $50 m \pm 5 m$ ); but the potential looseness around the interpretation of fifty meters in the comparative (5) seems not to involve such a default coarse interpretation, but rather relate to the maximum possible degree of precision compatible with a given context, which may be limited by factors such as measurement error.

In short, the flexibility to interpret round numbers at different levels of precision disappears when they are embedded in comparative statements.

b) Approximators in comparatives are NPIs. The idea that the comparative is in some way incompatible with variation in precision level receives initial support from the observation that overt approximators are disallowed in this position:

a. *Mabel owns more than about one hundred sheep.

b. *The meeting lasted more than roughly forty five minutes.

c. *The rope is more than exactly fifty meters long.

These are acceptable in reply contexts; for example, (6a) might be used to refute a prior claim that the number sheep she has is about one hundred. But in an out-ofthe-blue context, they are ill formed.

But surprisingly, while approximators cannot occur in the comparative in positive sentences, they are acceptable in the corresponding negative sentences, as well as other contexts that license negative polarity items, in particular if-clauses and the restrictor of universal quantifiers. For example:

a. Mabel owns no more than about one hundred sheep.

1 To my knowledge, the only previous mention of this pattern is in Sauerland \& Stateva 2011, where the observation is attributed to C. Cummins. The authors do not attempt an explanation. 
Imprecision

b. Mabel doesn't own more than about one hundred sheep.

c. I doubt Mabel owns more than about one hundred sheep.

d. If Mabel owns more than about one hundred sheep, we'll need to get more vaccine.

e. Every farmer who owns more than about one hundred sheep will benefit from the new legislation.

That is, approximators in comparatives appear to behave as negative polarity items.

To investigate how robust this pattern is, data were sourced from the Corpus of Contemporary American English (COCA; Davies 2008-), a 450-million word corpus spanning the years 1990-2012, and the following five genres: newspaper, magazine, fiction, academic texts and spoken language.

A search for the string "than about" followed by a numeral or number word yielded 330 relevant examples, involving not only the comparative more but also other comparative forms such as less, higher and larger; an additional 5 irrelevant tokens were excluded. As seen in Table 1, the pattern represented in (6)-(7) is to a large extent supported. The majority of tokens of than about $n$ occurred in negative contexts, a class that includes direct negation of the comparative element, sentential negation and indirect negation (as in examples (8a-c), respectively). Also represented were if-clauses and other conditional constructions, as in (9); universals, as in (10); and a variety of contexts that could be characterized as generic and/or definitional, as in (11).

a. All prior bull markets have ended at ratios no higher than about 35 to 1 .

b. The whole project wouldn't cost more than about two million dollars.

c. It's rare for horses to sleep for more than about 5 or 10 minutes at a time on the ground.

(9) If the potatoes are larger than about 2 inches in diameter, cut in halves ...

(10) Anybody younger than about 45 today had no direct connection to the Vietnam War.

(11) a. Norplant is somewhat less effective in women who weigh more than about 150 pounds.

b. Stage $\mathrm{I}$ is defined as a tumor less than about three-quarters of an inch across.

Other positive contexts, as in (12), accounted for only a small minority of tokens.

(12) a. The border with Canada stretches more than about 4,000 miles ...

b. At the time we put it on line we believed we had less than about five hours of consciousness remaining before the crew would have passed out. 


\begin{tabular}{l|rr|rr} 
& \multicolumn{2}{|c}{ than about $n$} & \multicolumn{2}{c}{ about $n$} \\
\hline & $\#$ & $\%$ & $\#$ & $\%$ \\
\hline Negative & 196 & 59 & - & - \\
Conditional & 41 & 12 & - & - \\
Universal & 22 & 7 & - & - \\
Generic & 48 & 15 & 3 & 2 \\
Other positive & 23 & 7 & 183 & 98 \\
\hline Total & 330 & 100 & 186 & 100 \\
\hline
\end{tabular}

Table 1 Distribution of about

Positive contexts were somewhat more frequent when the comparative form was less than (20\%) or another inherently negative element (13\%), but were still in the minority. As a point of comparison, out of a sample of 186 tokens of bare about $n$ (sourced by extracting from COCA a random sample of 200 tokens of about + number/number word and excluding irrelevant examples), virtually all occurred in positive contexts (see Table 1).

The data available via COCA are too sparse to allow a quantitative analysis of other approximators, but qualitatively the same distribution holds. The naturally occurring examples of low-precision approximators in (13), sourced via Google, likewise support this point: Their acceptability is degraded if the negative element is removed. It is less easy to find comparable examples with approximators signaling high precision (there are no tokens of this sort on COCA), but (14), also sourced via Google, demonstrates that a similar asymmetry is present here as well.

(13) a. A good quality rabbit pellet should have at least $22 \%$ crude fiber and $*$ (no) more than approximately $14 \%$ protein.

b. I *(don't) want to spend more than $\$ 50$ or so.

c. None/*some of the indicators rose more than roughly two percent.

(14) The station can handle *(no) more than exactly 8 trains per hour.

The restricted status of approximators in comparatives has not to my knowledge been previously noted, and is something that a comprehensive theory of numerical imprecision must account for.

\section{Theories of imprecision}

Two promising analyses of imprecision have been put forward in recent years. I present these theories in this section, and in the next section discuss their ability to account for the facts outlined above. 
Imprecision

\subsection{Pragmatic halos}

Lasersohn (1999) proposes a pragmatic theory of imprecision according to which speakers may utter sentences that, while strictly speaking false, are 'close enough' to true for practical purposes. An example is the assertion that The townspeople are asleep when the vast majority are sleeping but a small number are, exceptionally, awake. Closeness to the truth is modeled via pragmatic halos. Each expression of the language is assigned a denotation as usual, but this denotation is also associated with a set of entities of the same semantic type-its halo-which represent the values that differ from the denotation in only pragmatically ignorable ways. For example, three o'clock denotes some point in time $i$; its pragmatic halo is a set of times which in the given context are not meaningfully different from $i$.

$$
\begin{aligned}
& \text { a. } \llbracket \text { three o'clock } \rrbracket=i \\
& \text { b. } H_{C}(\text { three o'clock })=\{\ldots, g, h, i, k, l, \ldots\}
\end{aligned}
$$

Halos of complex expressions are derived compositionally from the halos of their constituents. A sentence may then be felicitously uttered if some element in its halo is true, even if it itself is not.

Approximators can also be analyzed via halos. Lasersohn proposes that those that specify a high level of precision, such as exactly, operate by reducing the halo of an expression to the minimum possible in the context. Approximators such as about that signal a low precision level have the not entirely parallel function of expanding the denotation of an expression to include the halo of the unmodified form. Lasersohn does not, however, work this out compositionally for approximators such as those in $(3 \mathrm{a}-\mathrm{c})$, and we will see below that doing so is not entirely unproblematic.

\subsection{Scale granularity}

Krifka (2009) develops an alternate analysis of imprecision based on the granularity of measurement scales. The central idea is that the results of measurement can be reported with respect to scales that differ in how coarse- or fine-grained they are, that is, in the size of their minimal units. Distance, for example, might be measured in terms of scales counting in kilometers, tens of kilometers, hundreds of kilometers, and so forth; number might be counted in units, tens, hundreds, etc. Coarser-grained scales have fewer values for representing measurements, and each of these values stands for a broader scalar range than values on a fine-grained scale. Thus the coarser the scale on which a numerical expression occurs, the more approximate interpretation it allows. Further pragmatic principles favor a numerical expression being interpreted relative to the coarsest scale on which it occurs, leading to the preference for-rather than just the availability of-the approximate interpretation. 
Krifka proposes that in our culture, scales are most commonly based on powers of 10 , or the result of applying operations of halving or doubling to such scales. By way of illustration, (16) represents four possible scales for the reporting of lengths:
a. $1 \mathrm{~m}-2 \mathrm{~m}-3 \mathrm{~m}-4 \mathrm{~m}-\ldots$
b. $10 \mathrm{~m}-20 \mathrm{~m}-30 \mathrm{~m}-40 \mathrm{~m}-\ldots$
c. $20 \mathrm{~m}-40 \mathrm{~m}-60 \mathrm{~m}-80 \mathrm{~m}-\ldots$
d. $50 \mathrm{~m}-100 \mathrm{~m}-150 \mathrm{~m}-200 \mathrm{~m}-\ldots$

In some domains, however, available granularity levels are based on other principles. Such is the case in time measurement, where the convention of dividing the day into 60-minute hours results in scales counting by hours, half hours, quarter hours, five-minute intervals and minutes. The above-discussed approximate interpretation of three o'clock thus might involve a scale of 5- or 15-minute granularity.

The mechanism of granularity has been implemented in several different ways: different granularity levels might be modeled as entirely distinct scales, as in (16); as probability distributions over a continuous scale (Krifka 2009); or via granularity functions that map points to intervals around them (Sauerland \& Stateva 2011). The latter authors show that a granularity-based approach also allows a natural account of the semantics of approximators. Granularity on their account is a contextual parameter of interpretation. Approximators can then be analyzed as resetting the level of granularity at which a numerical expression is interpreted: More-precise approximators such as exactly set the granularity parameter to the finest available in the context, whereas less-precise approximators such as approximately set it to the coarsest level.

\section{Comparing the theories}

Let us compare the two approaches outlined above, starting with the basic assumptions on which they are based, and then considering their ability to account for the data introduced in the previous sections.

\subsection{Assumptions}

Both of the above-described theories of imprecision have aspects that might be criticized as counterintuitive. In Lasersohn's theory of pragmatic halos (henceforth $\mathrm{PH})$, imprecision is-as the name suggests-a purely pragmatic phenomenon. An unwelcome consequence is that a large proportion of what we say using numerical expressions must be analyzed as strictly false. Events rarely if ever occur exactly at points of time such as 3:00 on the dot; probably no rope in the world is 50 meters 
Imprecision

long without a deviation of even a couple of millimeters; and so forth. Lasersohn considers this objection and ultimately rejects it, noting that it is still possible to make true assertions with number words (e.g., by using range expressions such as between 2:59 and 3:01). But we are still left with a formal notion of truth that is far removed from the intuitive one.

The scale granularity approach (henceforth SG) avoids this proliferation of falsity, in that a measure expression such as three o'clock has an interpretation on which it denotes a range around the precise point. But this too might cause us a bit of discomfort: It seems that we would want three o'clock at the most basic level to denote a single point in time, and even more clearly one hundred to denote the single value 100 . Thus this alternative is also not entirely satisfying from an intuitive perspective.

In terms of data that might help in adjudicating between these two positions, Lasersohn cites the contradictory nature of examples such as (17) as evidence for the falsity of imprecise usage (see also Lauer 2012 for a similar argument):

(17) \#Although Jane arrived at three o'clock, she didn't arrive until slightly after three o'clock.

While I agree that (17) is ill-formed, it is not clear that it demonstrates the falsity of the first clause in the event of an arrival time a few minutes before or after 3:00. Rather, much of the contradictory feel seems to stem from the presence of although, which itself establishes an opposition between the two clauses, and from the juxtaposition of at and slightly after. The following modified example, which introduces 3:02 instead as a precisification of three o'clock, is significantly improved.

(18) Jane arrived at three o'clock; more precisely, she arrived at 3:02.

Thus there seems at present to be no strong empirical evidence for one or the other of these views.

\subsection{Round vs. non-round numbers}

Turning to the data introduced above, SG has an advantage in accounting for the interpretive difference between round and non-round numbers. On Krifka's analysis, round numbers occur on coarser-grained scales than do non-round numbers, and thus allow more imprecise interpretations. We furthermore capture the gradient nature of roundness and the approximate interpretation it gives rise to. One hundred, for example, intuitively allows a rougher interpretation than does ninety. This follows directly from the conception of scales illustrated in (16): The coarsest scale on which 90 occurs is that counting in units of 10 , while 100 also occurs on coarser scales, for example those based on units of 20 and 50 . 
For PH, these same facts are problematic. Halos are defined in terms of values that differ from the denotation in only ways that are pragmatically ignorable in the context. Intuitively, 'ignorability' should be symmetric: If the difference between $a$ and $b$ is ignorable, then so too should be the difference between $b$ and $a$. But as discussed above, this is not what we observe in the imprecise use of measure terms: While three o'clock might be used for a time in a range that includes 3:02, the range for which three-oh-two can be used does not include 3:00. Thus the halo-based theory as formulated by Lasersohn does not offer any explanation for why some measure expressions-those that are round and/or correspond to salient higher-order units-allow more approximate interpretations than do others.

A potential route towards addressing this issue would be to augment Lasersohn's theory with findings from cognitive psychology that similarity judgments may be asymmetrical; as a well-known example, North Korea is judged more similar to China than China to North Korea (Tversky 1977). In a similar vein, one might predict 3:02 to be perceived as more similar to 3:00 than the reverse. But doing so would require a theory of which scalar points are salient in the relevant respect; in essence, it would presuppose a theory of granularity.

\subsection{Approximation and comparison}

The data introduced in Section 3 present further challenges for both analyses. Let us start with PH. On Lasersohn's account, halos of complex expressions are compositionally derived, in the default case via pointwise composition of the elements in the halos of their constituents. The halo of more than one hundred would thus be derived as a function of the halos of one hundred and more than. But if the context is such that the point 98 is in the halo of one hundred, then the halo of more than one hundred is expected to contain an element whose meaning could be characterized as ' $>98$ '. As discussed above, this is clearly not correct: more than one hundred cannot be felicitously used if the true value is 99 . To address this, we would need to stipulate that more than also has the effect of narrowing the halo of its complement to the narrowest one possible. This is essentially the analysis Lasersohn gives to exactly; but in the case of the comparative it would at best be ad hoc.

The facts relating to approximators in comparatives highlight a further issue with PH. Recall that expressions such as approximately and about are analyzed as expanding the denotation of an expression to include its halo. Suppose that one hundred denotes the point 100 on the number line. Then about one hundred would presumably come to denote some set of points centered on 100, which would lead to a type mismatch if it occurs in a position requiring a point-denoting expression. The most straightforward way to resolve this would be to introduce some sort of covert existential operator that picks an element out of the set in question. For simple 
Imprecision

examples this yields the correct result, as seen below (where for concreteness I adopt an implementation based on existential quantification over choice functions):

$\llbracket$ Mabel owns about one hundred sheep $\rrbracket^{C}=1$ iff

$\exists f$ [M. owns $f\left(H_{C}(100)\right)$ sheep]

But observe what happens in the case of approximators in negated comparatives, as in (7), repeated below. Crucially, (20a) means that the number of sheep Mabel owns is no more than the entire range that we would characterize as about one hundred, and thus no more than the maximum of this range. To capture this interpretation, the existential quantifier must take scope above rather than below negation, as in (20b); if the existential scopes instead under negation, as in (20c), we derive the incorrect reading that she owns no more than the minimum of that range:
a. Mabel owns no more than about one hundred sheep.
b. $\exists f\left[\neg\right.$ M. owns more than $f\left(H_{C}(100)\right)$ sheep ]
c. $\neg \exists f$ [ M. owns more than $f\left(H_{C}(100)\right)$ sheep ]

Wide-scope existential quantification may be plausible in this case, but becomes less so in more complex examples. In (21), the existential operator must scope out of the if clause. In (22), it must scope over the higher-clause negative verb. The LF in (22b) has the added issue that it seems to attribute to John a belief about a particular number within the halo of 100, whereas intuitively his doubt relates to some range as a whole that might be described as about one hundred.

(21) a. If Mabel owns more than about one hundred sheep, we'll need more vaccine.

b. $\exists f$ [ if M. owns more than $f\left(H_{C}(100)\right)$ sheep, we'll need more vaccine ]

(22) a. John doubts that Mabel owns more than about one hundred sheep.

b. $\exists f\left[\right.$ J. doubts that M. owns more than $f\left(H_{C}(100)\right)$ sheep ]

In summary, $\mathrm{PH}$ is not clearly able to derive the correct interpretation for approximators in the positions where they felicitously occur, to say nothing of the issue of how their restricted distribution might be accounted for.

With regards to these facts, SG again fares somewhat better. On their approximate interpretations, measure expressions such as one hundred and three o'clock denote scalar ranges as single units. More than one hundred, for example, can thus only be a value greater than some (perhaps trivial) range containing 100. The spurious low readings that were problematic for $\mathrm{PH}$ are never generated. Expressions including approximators such as about one hundred likewise denote ranges as units. This naturally yields the correct interpretations for examples such as (7), without the need 
to introduce additional mechanisms or stipulations. Mary owns no more than about one hundred sheep, for example, can only mean that she has no more than the entire range denoted by about one hundred; this is the desired result.

There are, however, other facts that are more problematic for SG. We first of all require an explanation for the observation that the comparative necessarily selects for a maximally fine granularity level. A further complication is that while the semantic interpretation of comparatives is necessarily precise, granularity effects are nonetheless observed at a pragmatic level. Specifically, Cummins, Sauerland \& Solt (2012) provide experimental evidence that examples such as (23) give rise to scalar implicatures that are granularity dependent.

(23) a. More than 100 people attended the meeting.

b. More than 110 people attended the meeting.

From (23a) participants tend to infer 'not more than 150' or 'not more than 200', while a typical inference from (23b) is 'not more than 120'. This can be explained as an implicature calculated relative to the next highest point on a scale of the same granularity level at which the modified numeral can be interpreted. In the (a) example above, this might be a a scale of granularity 50 or 100, in the (b) example, one of granularity 10. Thus it seems that two levels of granularity are active at once: a fine-grained level at which the semantic interpretation is derived, and a second coarser-grained one involved in the calculation of pragmatic inferences. It is not obvious how this could be implemented within the existing models of granularity discussed above.

Finally, SG-like PH-offers no immediate explanation for the NPI-like behavior of approximators in comparatives.

\section{Proposal}

In what follows I adopt the scale granularity approach, and seek to give it a new implementation that overcomes the issues highlighted above.

The starting point for my analysis is the metaphor of the ruler as measuring instrument (Figure 1). A ruler is itself a continuous extent, but a discrete structure is imposed on it via a hierarchical system of markings of different degrees of prominence, which make it possible to perform measurement at varying levels of precision. In the example depicted here, for instance, we could choose to measure lengths in inches, in half inches, in quarter inches, or in eighth inches. In each case, the measurement that will be reported is that corresponding to the closest mark at the chosen level. I propose that this gives us a clue as to the correct view of granularity as it pertains to natural language measure expressions.

The proposal consists of several steps, which I outline below. 
Imprecision

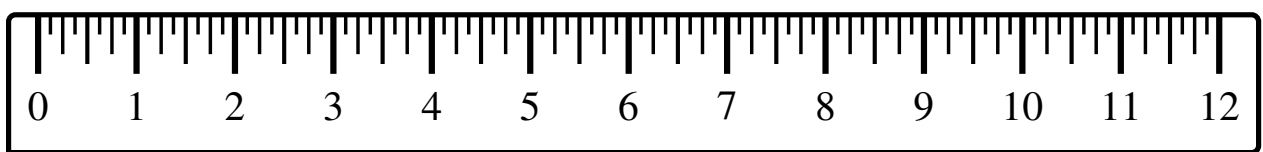

Figure 1 The ruler as a model of scale granularity

Point denotations for measure expressions. I assume a degree-based semantic framework in which measure expressions denote degrees (type $d$ ), conceptualized here as points on a maximally fine-grained scale. In the case of number words, the relevant scale is the positive integers, or perhaps the rational or real numbers (see Fox \& Hackl 2006). In the case of other sorts of measure expressions, it is the scale associated with some other dimension of measurement. For example:
a. $\llbracket$ one hundred $\rrbracket=100$
b. $\llbracket$ three o'clock $\rrbracket=3: 00$

This contrasts with previous granularity-based analyses in which measure expressions have interpretations on which they denote scalar ranges.

Granularity as alternatives. Granularity may now be represented in terms of sets of alternatives to a measure expression. We start with some standard unit gran, which defines a standard sequence $S_{\text {gran }}$. In (25)-(27) I give examples of standard sequences for the measurement of number, length and duration, respectively:

$$
\begin{aligned}
& \text { a. } \operatorname{gran}=10 ; S_{10}=\{10,20,30, \ldots\} \\
& \text { b. } \operatorname{gran}=20 ; S_{20}=\{20,40,60, \ldots\} \\
& \text { gran }=5 \mathrm{~m} ; S_{5 m}=\{5 \mathrm{~m}, 10 \mathrm{~m}, 15 \mathrm{~m}, \ldots\} \\
& \text { gran }=15 \mathrm{~min} ; S_{15 \min }=\{15 \min , 30 \mathrm{~min}, 45 \min , \ldots\}
\end{aligned}
$$

Per the earlier discussion, typical choices for gran are powers of 10 and the results of halving and doubling these. In some cases, domain specific measurement conventions also come into play, as in (27).

For a measure expression $\alpha$ and granularity level gran, a set of alternatives to $\alpha$ can then be defined as in (28); an example is given in (29).

$$
\operatorname{ALT}_{\text {gran }}(\alpha)=\left\{\alpha^{\prime}: \llbracket \alpha^{\prime} \rrbracket \in S_{\text {gran }}\right\}
$$

I take $A L T_{\text {gran }}(\alpha)$ to be undefined if $\llbracket \alpha \rrbracket$ is not in $S_{\text {gran }}$; that is, a measure expression only has alternatives at granularity levels in which its denotation participates. 
The members of $A L T_{\text {gran }}$ can be likened to the markings on the ruler: They represent the (only) choices the speaker has to report a measurement at granularity level gran.

Truth relative to granularity level. We come now to the crucial stage. I propose that the truth or falsity of a sentence containing a measure expression is relativized to the granularity level at which that expression is interpreted. For each measure expression, the corresponding granularity level gran is contextually determined via an assignment function $g$. Truth at a granularity level is then defined in terms of the scalar distance that the actual exact measure would need to be displaced in order to achieve truth under a perfectly precise $($ gran $=0)$ interpretation. Formally:

(30) For a sentence $\phi$ containing a measure expression $\alpha, \llbracket \phi \rrbracket^{g}=1$ iff there is no $\alpha^{\prime} \in A L T_{\text {gran }}(\alpha)$ such that $\llbracket \phi\left[\alpha^{\prime} / \alpha\right] \rrbracket^{g[\operatorname{gran}=0]}=1$ would require a smaller scalar displacement of the actual measure than $\llbracket \phi \rrbracket^{g[\text { gran }=0]}=1$.

In simple terms, a proposition containing a measure expression is thus evaluated as true iff there is no better choice of expression at the relevant granularity level.

Let us observe how this works in some concrete examples. Consider the two sentences in (31) in the situations depicted in (32), where we assume that the granularity level assigned by $g$ to the measure expression fifty meters is gran $=5 \mathrm{~m}$.

a. The rope is fifty meters long.

b. The rope is more than fifty meters long.

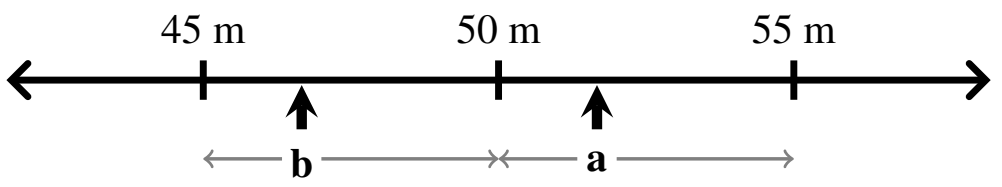

Starting with (31a), the replacement of fifty meters with members of $A L T_{5 m}$ (fifty meters) yields sentences including the following to be evaluated at gran $=0$ :
a. «the rope is forty-five meters long $\rrbracket^{g[\operatorname{gran}=0]}$
b. 【the rope is fifty meters long $\rrbracket^{g[\text { gran }=0]}$
c. 【the rope is fifty-five meters long $\rrbracket^{g[\text { gran }=0]}$

If the actual length of the rope is that depicted as (a) in (32), the original (31a) will be evaluated as true, because ( $33 \mathrm{~b}$ ) is the alternative whose truth would require the smallest scalar displacement of this measure. But if the rope's actual length is (b), the sentence will be evaluated as false, since there is a better expression choice at 
Imprecision

this granularity level: The truth of (33a) requires a smaller displacement of the actual measure.

Crucially, a different pattern obtains for the comparative in (31b). In this case, the sentential alternatives that are derived include the following:

a. [the rope is more than forty-five meters long $\rrbracket^{g[\text { gran }=0]}$

b. 【the rope is more than fifty meters long $\rrbracket^{g[\operatorname{gran}=0]}$

c. 【the rope is more than fifty-five meters long $\rrbracket^{g[\text { gran }=0]}$

In situation (a), or any other case in which the actual measure is greater than exactly $50 \mathrm{~m}$, (31b) will be evaluated as true, because (34b) is already true at gran $=0$ with no displacement of the actual value needed. Conversely, it will be evaluated as false in situation (b), or any other case of actual measure $\leq 50 \mathrm{~m}$, because there are other alternatives for which it is already true at gran $=0$; in situation (b), for example, (34a) is such an alternative. Furthermore, this is the case regardless of how gran is set contextually: (31b) is true in all and only those cases that the actual measure is greater than (exactly) 50 meters, and false otherwise. The result is a necessarily precise interpretation for the measure expression.

Note that in the formulation above, the scalar zero, and expressions that denote it, do not participate in granularity-based alternative sets. This is intended to capture the intuition that expressions such as zero sheep cannot be used imprecisely. Initially this choice appears to create an issue with the reporting of low scalar values: We predict that at the 5-meter granularity level, both the rope is five meters long and the rope is more than five meters long could be truthfully uttered in the case of a 1-meter-long rope, as there is no better expression at that granularity level that could be used instead. I propose that this is ruled out by a constraint on the appropriate choice of granularity level, namely that a granularity level gran is only felicitous for the reporting of values on the order of gran and above. A 5-meter granularity level is thus simply not appropriate to describe the length of a 1-meter-long rope.

Approximators as granularity functions. As the final step, we require a semantics for approximators. To this end, observe that (30) has the effect of associating measure expressions with scalar segments whose widths are constrained by the prevailing granularity level. I propose that this is lexicalized by approximators, which map points to intervals around them. The general template for approximator semantics is the following:

$$
\llbracket \text { APPROXIMATOR } \mathrm{n} \rrbracket^{g}=\left(n-\operatorname{gran}^{\prime} / 2, n+\operatorname{gran}^{\prime} / 2\right)
$$

That is, the range denoted by an expression containing an approximator is bounded by the midpoints between two sequential elements of $S_{g r a n^{\prime}}$ for some gran'. In 
the case of approximators of lower precision such as about and roughly, gran' is selected as the coarsest possible choice for gran in the given context. In the case of the high precision exactly, it is the finest possible choice in the context. Thus for example about 50 meters might come to denote the range $50 m \pm 5 m$, while exactly $50 \mathrm{~m}$ denoted the narrower range $50 \mathrm{~m} \pm 0.5 \mathrm{~cm}$.

Crucially, I take the scalar ranges derived in this way to be interpreted as indivisible wholes. Formally, they can be viewed as coarse-grained degrees (type $d$ ). This represents a departure from the more typical view of degrees as points, and also from existing theories that treat degrees as intervals (e.g., Kennedy 1997; Schwarzschild \& Wilkinson 2002). The proposal here is that we have both sorts of degrees: points denoted by unmodified measure expressions and intervals denoted by their approximator-modified counterparts.

As in previous implementations of the granularity-based approach, this analysis of approximators yields the correct results for their occurrences in comparatives. For example, if gran' $^{\prime}$ is set to 10 , about one hundred denotes the degree constituted by the interval $(95,105)$. More than about one hundred then corresponds to the scalar range exceeding the maximum of this interval, i.e., the range $\geq 105$. Conversely, no more than about one hundred corresponds to the range not exceeding the maximum of this interval. Per the discussion in Section 4.3, this is the desired result.

Observe that there is once again an interesting consequence relating to the zero point on a scale. Per (35), the interval derived via combination with an approximator is symmetrical around the original point. In the case of a scalar zero, the only interval of this form is the trivial one. Composition with any approximator is then predicted to have a trivial effect. This can be taken to account for the infelicity of examples such as about/roughly/approximately/exactly zero sheep, in contrast to about/roughly/approximately/exactly zero degrees Celsius, where zero does not refer to a scalar endpoint.

Let us summarize what has been achieved in relation to the issues discussed earlier in the paper. First, the analysis developed in this section avoids some of the more unintuitive aspects of previous accounts (cf. the discussion in Section 4.1). Measure expressions are not ambiguous, but rather always denote points on scales. But sentences in which they occur may nonetheless be evaluated as true (and not only "close enough" to true) if the actual measure deviates from that point. We further derive approximate interpretations for round numbers in simple sentences, while at the same time blocking this possibility for the same expressions in comparatives, something that is not achieved by previous analyses. Finally, we derive the correct semantic interpretation for expressions containing approximators, including when these occur in comparative constructions. In the next section I turn to some still open questions. 
Imprecision

\section{Structurally defined alternatives and blocking effects}

The analysis developed above relies crucially on the conceptualization of granularity in terms of sets of alternatives. To account for the remainder of the data introduced in Sections 2-4, and in particular for the NPI status of approximators in comparatives, we need to extend the account by considering a wider class of alternatives to a sentence in which a measure expression occurs.

To this end, I adopt Katzir's (2007) theory of structurally defined alternatives, developed originally for the analysis of scalar implicatures. According to Katzir's account, the alternatives to a structure $\phi$ that affect its interpretation are derived structurally via substitution and deletion. The choice between alternatives is governed by a conversational principle that is neo-Gricean in spirit. I assume the following version of this principle (where $\operatorname{ALTS}(\phi)$ is the set of structural alternatives to $\phi$ ):

Conversational principle: Do not assert $\phi$ if there is another sentence $\phi^{\prime} \in$ $\operatorname{ALTS}(\phi)$ such that both: a) $\phi^{\prime}$ is 'better than' $\phi$; and b) $\phi^{\prime}$ is weakly assertable.

A sentence $\phi$ is weakly assertable if the speaker believes it to be true, relevant and supported by the evidence. Following discussion by Katzir, I take 'better than' to be defined in terms of two component factors: informativity (in a sense that will be elaborated below) and simplicity.

Katzir shows that this view of alternatives can account for a variety of implicature types, including both scalar implicatures and ignorance implicatures. I propose here that it also provides an explanation for the restricted distribution of approximators in comparatives. The idea that I pursue is that in some (but not other) of the contexts in which these expressions might occur, there is always a better alternative that is weakly assertable, with the result that the corresponding sentence is blocked.

Let us begin with the ungrammatical example $\phi$ in (37a). Deletion of about allows us to derive $\phi^{\prime}$ in (37b) as an alternative.

a. $\phi=*$ Mabel owns more than about one hundred sheep.

b. $\phi^{\prime}=$ Mabel owns more than one hundred sheep.

- $\phi^{\prime}$ weakly assertable whenever $\phi$ is

-simplicity: $\phi^{\prime} \succ \phi$

-informativity: ?

Observe first that according to the analysis developed in the previous section, the scalar range describable as more than about one hundred is properly contained in that describable as more than one hundred (this is depicted graphically in Figure 2). Thus $\phi$ entails $\phi^{\prime}$, and as such any speaker who is in a position to assert $\phi$ could also assert $\phi^{\prime}$. The alternative $\phi^{\prime}$ furthermore has an advantage in terms of simplicity. 


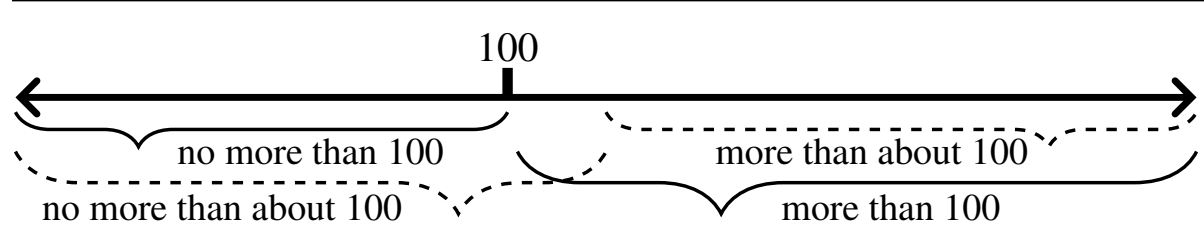

Figure 2 Scalar ranges corresponding to comparative statements

When it comes to informativity, the relative ranking of the two is less obvious. Katzir, like previous authors, construes informativity in terms of asymmetrical entailment, and in this sense $\phi$ clearly has the advantage, for the reason just described. But there is another sense in which $\phi$ is actually less informative than $\phi^{\prime}$, namely that unlike its simpler alternative, it fails to have sharply defined truth conditions. The lower bound for more than about one hundred depends on the choice of the granularity level $\mathrm{gran}^{\prime}$ that determines the denotation of about one hundred, and any situation of utterance may be compatible with multiple possibilities. This contrasts with $\phi^{\prime}$, where 100 constitutes a sharp dividing line between what does and does not count as 'more than 100'. I propose that these two aspects of informativityscalar strength and lack of vagueness-cancel one another, leaving neither of the two alternatives in (37) with a clear advantage over the other. This coupled with the simpler structure of $\phi^{\prime}$ makes the latter better in the overall sense. The consequence is that there is never a situation in which $\phi$ can be asserted by a cooperative speaker.

The situation is different in the negated comparative in (38):

a. $\phi=$ Mabel owns no more than about one hundred sheep.

b. $\phi^{\prime}=$ Mabel owns no more than one hundred sheep.

-informativity: $\phi^{\prime} \sim \phi$

-simplicity: $\phi^{\prime} \succ \phi$

Here $\phi^{\prime}$ is again the better alternative overall, being simpler than $\phi$ and no less informative. But in this case the entailment relation between the two is reversed (see again Fig. 2). The consequence is that there are situations in which $\phi$ is assertable while $\phi^{\prime}$ is not; thus in this case no blocking occurs. From a speaker's use of $\phi$, the hearer is then entitled to infer that such a situation obtains, i.e., that it is not the case that the speaker believes $\phi^{\prime}$ to be true, relevant and supported by the evidence. A particular inference that might be drawn is that the speaker does know whether or not 'no more than one hundred' obtains. This is exactly the feel that examples such as (38a) give: The speaker is hedging his or her claim, by allowing the possibility that the number is close to, and perhaps slightly above, 100.

Observe that a similar sort of explanation also applies to the other contexts in which approximators in comparatives felicitously occur, including if clauses, 
Imprecision

universals and generics (see (7) and (10)-(11) above). All of these express rules or generalizations of some sort. The inclusion of the approximator results in a weaker statement than would obtain with an unmodified numeral, making the resulting sentence an appropriate choice for a speaker seeking to hedge his/her assertion.

Recall finally from Section 4 that comparatives such as "more than one hundred' give rise to granularity-based scalar implicatures; for example, more than one hundred tends to imply 'not more than 150'. This too can be explained within a structure-based view of alternatives. We must simply add the requirement that in the case of a sentence $\phi$ containing a measure expression $\alpha$, substitution for $\alpha$ is constrained to members of $A L T_{\text {gran }}(\alpha)$ for some granularity level gran, and furthermore that all members of $A L T_{\text {gran }}(\alpha)$ are considered as equivalent in simplicity.

Taking $\phi$ in (39a) as an example, if gran is set to 50, then the permissible substitution of one hundred fifty in place of one hundred yields (39b) as an alternative.

a. $\phi=$ Mabel owns more than one hundred sheep.

b. $\phi^{\prime}=$ Mabel owns more than one hundred fifty sheep.

-informativity: $\phi^{\prime} \succ \phi$

-simplicity: $\phi^{\prime} \sim \phi$

$\phi^{\prime}$ is more informative than $\phi$, and no more complex; in total, it is a better alternative. From a speaker's assertion of $\phi$, a hearer is thus licensed to infer that $\phi^{\prime}$ is not weakly assertable. Assuming the extra information conveyed by $\phi^{\prime}$ would be relevant, we derive the familiar sort of scalar implicature, though one conditioned by granularity, namely that the speaker doesn't believe Mabel to have more than 150 sheep.

\section{Concluding remarks on granularity}

In this paper, I have developed an account of numerical imprecision based on two distinct types of alternatives. First, granularity itself is conceptualized in terms of a set of alternatives to a measure expression; this set plays a role similar to that of the lines on a ruler, allowing an expression to be used truthfully to report a measurement if it is the best choice at the relevant granularity level. Second, the restricted distribution of certain measure expressions is analyzed as deriving from competition with structurally simpler alternatives that the speaker might have uttered.

An open question is whether this analysis can be extended to other varieties of imprecision, and other facts that have been analyzed via the mechanisms applied to the numerical variety. These include the imprecise use of absolute gradable adjectives such as full, the semantics of degree modifiers such as slightly and completely (Sassoon 2012), and phenomena such as metalinguistic comparison (Morzycki 2011). The central issue is what the relevant alternatives would be in these cases. 


\section{References}

Cummins, Chris, Uli Sauerland \& Stephanie Solt. 2012. Granularity and scalar implicature in numerical expressions. Linguistics and Philosophy 35(2). 135169. doi:10.1007/s10988-012-9114-0.

Davies, Mark. 2008-. The Corpus of Contemporary American English (COCA): 450 million words, 1990-present. Available online at http://corpus.byu.edu/coca/.

Fox, Danny \& Martin Hackl. 2006. The universal density of measurement. Linguistics and Philosophy 29(5). 537-586. doi:10.1007/s10988-006-9004-4.

Katzir, Roni. 2007. Structurally-defined alternatives. Linguistics and Philosophy 30. 669-690. doi:10.1007/s10988-010-9074-1.

Kennedy, Christopher. 1997. Projecting the adjective: The syntax and semantics of gradability and comparison: University of California at Santa Cruz Ph.D. dissertation.

Krifka, Manfred. 2002. Be brief and be vague: and how bidirectional optimality theory allows for verbosity and precision. In David Restle \& Dietmar Zaefferer (eds.), Sounds and Systems. Studies in Structure and Change: A Festschrift for Theo Vennemann, 439-458. Berlin: Mouton de Gruyter.

Krifka, Manfred. 2009. Approximate interpretations of number words: A case for strategic communication. In Erhard W. Hinrichs \& John Nerbonne (eds.), Theory and Evidence in Semantics, 109-132. Stanford: CSLI Publications.

Lasersohn, Peter. 1999. Pragmatic halos. Language 75(3). 522-551.

Lauer, Sven. 2012. On the pragmatics of pragmatic slack. In Ana Aguilar Guevara, Anna Chernilovskaya \& Rick Nouwen (eds.), Sinn und Bedeutung 16, vol. 2, 389-402. MIT Working Papers in Linguistics.

Morzycki, Marcin. 2011. Metalinguistic comparison in an alternative semantics for imprecision. Natural Language Semantics 19. 39-86. doi:10.1007/s11050-0109063-5.

Sassoon, Galit. 2012. A slightly modified economy principle: stable properties have non-stable standards. In Evan Cohen (ed.), Israel Association of Theoretical Linguistics (IATL) 27, 163-182. MIT Working Papers in Linguistics.

Sauerland, Uli \& Penka Stateva. 2011. Two types of vagueness. In Paul Égré \& Nathan Klinedinst (eds.), Vagueness and Language Use, 121-145. Houndmills, Basingstoke: Palgrave Macmillan.

Schwarzschild, Roger \& Karina Wilkinson. 2002. Quantifiers in comparatives: A semantics of degree based on intervals. Natural Language Semantics 10. 1-41. doi:10.1023/A:1015545424775.

Tversky, Amos. 1977. Features of simlarity. Psychological Review 84. 327-352. doi:10.1037/0033-295X.84.4.327. 
Imprecision

Stephanie Solt

Centre for General Linguistics (ZAS)

Schützenstr. 18

10117 Berlin

Germany

stephanie.solt@gmail.com 